\title{
Universality of Squashed-Sphere Partition Functions
}

\author{
Pablo Bueno, ${ }^{1}$ Pablo A. Cano, ${ }^{2}$ Robie A. Hennigar, ${ }^{3}$ and Robert B. Mann ${ }^{3}$ \\ ${ }^{1}$ Instituut voor Theoretische Fysica, KU Leuven, Celestijnenlaan 200D, B-3001 Leuven, Belgium \\ ${ }^{2}$ Instituto de Física Teórica UAM/CSIC, C/ Nicolás Cabrera,13-15, C.U. Cantoblanco, 28049 Madrid, Spain \\ ${ }^{3}$ Department of Physics and Astronomy, University of Waterloo, Waterloo, Ontario, Canada N2L $3 G 1$
}

(Received 27 August 2018; revised manuscript received 15 January 2019; published 20 February 2019)

We present several results concerning the free energy of odd-dimensional conformal field theories (CFTs) on squashed spheres. First, we propose a formula which computes this quantity for holographic CFTs dual to higher-curvature gravities with second-order linearized equations of motion. As opposed to standard on-shell action methods for Taub geometries, our formula only involves a simple evaluation of the corresponding bulk Lagrangian on an auxiliary pure anti-de Sitter (AdS) space. The expression is closely related to the function determining the possible AdS vacua of the bulk theory in question, which we argue to act as a generating functional from which correlation functions of the boundary stress tensor can be easily characterized. Finally, based on holographic results and free-field numerical calculations, we conjecture that the subleading term in the squashing-parameter free-energy expansion is universally controlled by the stress-tensor three-point function charge $t_{4}$ for general $(2+1)$-dimensional CFTs.

DOI: 10.1103/PhysRevLett.122.071602

Euclidean conformal field theories (CFTs) coupled to background fields can be used to learn important lessons about the dynamics of the theory in question. A prototypical example corresponds to supersymmetric CFTs, where localization techniques have allowed for notable progress-see, e.g., Ref. [1]. For nonsupersymmetric theories, a natural possibility consists in coupling the theory to curved background metrics. This approach has produced some exact and universal results valid for general CFTs $[2,3]$ and has found various applications, e.g., in holographic cosmology [4-7]. Particularly interesting is the case of spherical backgrounds, whose partition functions-equivalently, free energies: $F_{\mathbb{S}^{d}}=$ $-\log \left|Z_{\mathbb{S}^{d}}\right|$-have been conjectured to be renormalizationgroup monotones for general odd-dimensional QFTs [8-10].

In this Letter, we will consider CFTs on deformed spheres and study the effect that such deformations have on $F$. The focus will be on a particular class of squashed spheres, $\mathbb{S}_{\varepsilon}^{d}$, which preserve a large subgroup of isometries of the round ones [11]. In particular, they are characterized by being Hopf fibrations over the complex projective space $\mathbb{C P}^{k}[k \equiv(d-1) / 2]$, namely, $\mathbb{S}^{1} \hookrightarrow \mathbb{S}_{\varepsilon}^{d} \rightarrow \mathbb{C P}^{k}$. The metric on these squashed spheres is given by

Published by the American Physical Society under the terms of the Creative Commons Attribution 4.0 International license. Further distribution of this work must maintain attribution to the author(s) and the published article's title, journal citation, and DOI. Funded by SCOAP .

$$
d s_{\mathbb{S}_{\varepsilon}^{d}}^{2}=\frac{d s_{\mathbb{C} \mathbb{P}^{k}}^{2}}{(d+1)}+(1+\varepsilon)\left(d \psi+\frac{A_{\mathbb{C P}^{k}}}{(d+1)}\right)^{2}
$$

where $\psi$ is a periodic coordinate which parametrizes the $\mathbb{S}^{1}, d s_{\mathbb{C P}^{k}}^{2}$ is the Einstein metric on $\mathbb{C P}^{k}$ normalized so that $R_{i j}=g_{i j}$, and $J=d A_{\mathbb{C P}^{k}}$ is the Kähler form on $\mathbb{C P}^{k}$. The parameter $\varepsilon$ measures the degree of squashing of the sphere and, in principle, it can take values in the domain $\varepsilon \in(-1,+\infty)$, the round sphere corresponding to $\varepsilon=0$. In $d=3$, which is the simplest case, $\mathbb{C P}^{1} \cong \mathbb{S}^{2}$, and we can write $d s_{\mathbb{S}^{2}}^{2}=d \theta^{2}+\sin ^{2} \theta d \phi^{2}, \quad A_{\mathbb{S}^{2}}=2 \cos \theta d \phi$ in standard spherical coordinates.

This class of squashed spheres can be easily studied holographically [12-17], as the relevant bulk geometries belong to the well-known anti-de Sitter (AdS)-Taub-NUT and bolt family. Our first main result—see Eq. (9)—is a universal formula for the free energy of a broad class of holographic CFTs on squashed spheres. The formula is automatically UV finite and, in fact, does not require knowing the corresponding NUT solutions explicitly. It holds for higher-curvature bulk theories with second-order linearized equations of motion, correctly reproducing all known results available for such theories, and passes several consistency checks coming from field theory considerations. Our second result—see Eq. (12)—is an expression for the subleading term in the small squashingparameter expansion of $F_{\mathbb{S}_{\varepsilon}^{d}}$, which, based on holographic and free field calculations, we conjecture to be controlled by the stress-tensor three-point function coefficient $t_{4}$ for general CFTs. As an additional consequence of our results in the holographic context, we observe that, for the class of 
bulk theories just described, the function that determines the possible AdS vacua of the theory-see Eq. (4)—acts as a generating functional for the boundary stress tensor, in the sense that we can easily characterize its correlators by taking trivial derivatives of such function, drastically simplifying the standard holographic calculations-see Eqs. (5), (16), (17), and (18).

Higher-order gravities and holography on squashed spheres.-AdS and CFT [18-20] provides a powerful playground for exploring the physics of strongly coupled CFTs. In some cases, the possibility of mapping intractable field-theoretical calculations into manageable ones involving gravity techniques allows for the identification of universal properties valid for completely general CFTs. In this context, higher-curvature gravities turn out to be very useful, as they define holographic toy models for which many explicit calculations can be performed explicitly. The idea is that, if a certain property is valid for general theories, it should also hold for these models. This approach has been successfully used before, e.g., in the identification of monotonicity theorems in various dimensions [21,22], or in the characterization of entanglement entropy universal terms [23-26]. Naturally, particular higher-curvature interactions generically appear as stringy corrections to the effective actions of top-down models admitting holographic duals [27]. For the purposes just described, however, it is more useful to consider bulk models that are particularly amenable to holographic calculations-see, e.g., Refs. [28-33].

The Lagrangian of such kind of models can be generally written, in $(d+1)$ bulk dimensions, as

$$
\mathcal{L}=\frac{1}{16 \pi G}\left(\frac{d(d-1)}{L^{2}}+R+\sum_{n=2} \mu_{n} L^{2(n-1)} \boldsymbol{R}_{(n)}\right),
$$

where $L$ is some length scale, $G$ is Newton's constant, the $\mu_{n}$ are dimensionless couplings, and the $\boldsymbol{R}_{(n)}$ stand for the higher-curvature terms, constructed from linear combinations of order- $n$ curvature invariants. The AdS vacua of any theory of the form [Eq. (2)] can be obtained by solving [34]

$h\left(f_{\infty}\right) \equiv \frac{16 \pi G L^{2}}{d(d-1)}\left[\mathcal{L}\left(f_{\infty}\right)-\frac{2 f_{\infty}}{(d+1)} \mathcal{L}^{\prime}\left(f_{\infty}\right)\right]=0$,

where $\mathcal{L}\left(f_{\infty}\right)$ is the on-shell Lagrangian on pure $\operatorname{AdS}_{(d+1)}$ with radius $L / \sqrt{f_{\infty}}$. This can be easily obtained evaluating all Riemann tensors in Eq. (2) as $R_{a b c d}=$ $-f_{\infty} / L^{2}\left(g_{a c} g_{b d}-g_{a d} g_{b c}\right)$. Also, $\mathcal{L}^{\prime}\left(f_{\infty}\right) \equiv d \mathcal{L}\left(f_{\infty}\right) / d f_{\infty}$. It is easy to argue that the $\mathfrak{R}_{(n)}$ can always be normalized so that the function $h\left(f_{\infty}\right)$ in Eq. (3) reduces to the form [35]

$$
h\left(f_{\infty}\right)=1-f_{\infty}+\sum_{n=2} \mu_{n} f_{\infty}^{n} .
$$

For Einstein gravity, one just finds $f_{\infty}=1$, and the action scale $L$ coincides with the AdS radius.

We further restrict Eq. (2) to the particular subclass of theories whose linearized equations on maximally symmetric backgrounds are second order [36]. This subclasswhich we shall refer to as Einstein like [34]—contains infinitely many theories and includes the following: all Lovelock [37,38] and some $f$ (Lovelock) theories [39], Quasitopological gravity [40,41] and its higher-curvature extensions [42,43], Einsteinian cubic gravity in general dimensions [44], and generalized quasitopological gravity [45], among others [46-48]. The vast majority of all known theories of the form of Eq. (2) admitting nontrivial black hole and Taub solutions belong to this class.

As we show here, the function $h\left(f_{\infty}\right)$ contains a surprisingly great deal of additional nontrivial information for Einstein-like theories. First, given one such theory, it determines the effective gravitational constant through $G_{\text {eff }}=-G / h^{\prime}\left(f_{\infty}\right)$-see the Supplemental Material [49] for a proof. From the dual CFT point of view, this translates into the following relation with the charge $C_{T}$, which fully characterizes the CFT stress-tensor two-point function $[50,52]$

$$
C_{T}=-h^{\prime}\left(f_{\infty}\right) C_{T}^{E},
$$

where $C_{T}^{E}$ stands for the Einstein gravity result [54]

$$
C_{T}^{E}=\frac{\Gamma[d+2]\left(L / \sqrt{f_{\infty}}\right)^{d-1}}{8 \pi^{(d+2 / 2)}(d-1) \Gamma[d / 2] G} .
$$

In AdS/CFT, the semiclassical partition function is exponentially dominated by the bulk geometry with the smallest on-shell action satisfying the appropriate boundary conditions. Hence, the free energy of the CFT can be accessed from the regularized on-shell action of the bulk theory evaluated on the corresponding gravity solution [55]. When the boundary geometry is a squashed sphere of the form [Eq. (1)], the relevant bulk solutions are of the so-called Euclidean Taub-NUT and bolt class $[12,14,15]$. Such solutions are characterized by the NUT charge $n$ which, on general grounds, holography maps to the squashing parameter of the boundary geometry $\varepsilon$ through

$$
\frac{n^{2}}{L^{2}}=\frac{(1+\varepsilon)}{(d+1) f_{\infty}} .
$$

Naturally, constructing Taub solutions is a more challenging task than classifying the vacua of the theory and, in fact, only a few examples of such solutions have been constructed for Einstein-like Lagrangians of the form [Eq. (2)]. The simplest instances in $d=3$ correspond to Einsteinian cubic gravity [56], whose Lagrangian is given by [44] 


$$
\mathcal{L}^{\mathrm{ECG}}=\frac{1}{16 \pi G}\left[\frac{6}{L^{2}}+R-\frac{\mu L^{4}}{8} \mathcal{P}\right]
$$

where $\mathcal{P}=12 R_{a b}^{c d} R_{c d}^{e f} R_{e f}^{a b}+R_{a b}^{c d} R_{c d}^{e f} R_{e f}^{a b}-12 R_{a b c d} R^{a c} R^{b d}+$ $8 R_{a}^{b} R_{b}^{c} R_{c}^{a}$ is a new cubic invariant and $\mu$ is a dimensionless coupling. In $d \geq 5$, analytic Taub solutions have been constructed for Einstein [57] and Einstein-Gauss-Bonnet gravity [58-60] and there have been a number of holographic applications of these solutions [61-64]. Very recently, additional solutions have been discovered for other Einstein-like theories (both in $d=3$ and $d=5$ ) in Ref. [56].

In all these cases, the thermodynamic properties of the solutions can be accessed analytically. In particular, the computation of regularized on-shell actions can be performed after the introduction of various boundary terms and counterterms which account for the various UV divergences [14,33,65-68]. As long as the solution is the dominant saddle, the resulting on-shell action computes the free energy of the dual theory on a squashed sphere $\mathbb{S}_{\varepsilon}^{d}$. For sufficiently small $\varepsilon$, the relevant saddle is generically of the NUT type.

A universal formula for holographic squashed-spheres free energy.-Rather strikingly, we observe that the following simple pattern holds in all cases: the finite (and only physically meaningful) contribution to the free energy of a holographic CFT dual to an Einstein-like higher-order gravity theory on a squashed $\mathbb{S}_{\varepsilon}^{d}$ can be obtained by evaluating the on-shell Lagrangian of the corresponding theory on pure $\operatorname{AdS}_{(d+1)}$. The dependence on the squashing parameter appears encoded in the $\mathrm{AdS}$ radius of this auxiliary geometry, which is given by $L \sqrt{(1+\varepsilon) / f_{\infty}}$. Explicitly, we claim that for any theory of this kind the following formula holds

$$
F_{\mathbb{S}_{\varepsilon}^{d}}=(-1)^{(d-1) / 2} \frac{\pi^{(d+2) / 2}}{\Gamma\left[\frac{d+2}{2}\right]} \frac{\mathcal{L}\left[f_{\infty} /(1+\varepsilon)\right] L^{d+1}}{\left[f_{\infty} /(1+\varepsilon)\right]^{(d+1) / 2}} .
$$

This expression is drastically simpler than the standard onshell action approach, which relies on various theorydependent (boundary and counter) terms to extract this finite piece. Instead, in Eq. (9) the regularization is automatically implemented, and allows us to perform a general theory-independent analysis of the free energy of holographic CFTs on squashed spheres.

First, note that if we set $\varepsilon=0$, we recover the result for the free energy of the theory on a round $\mathbb{S}^{d}$, which plays a crucial role in establishing monotonicity theorems, particularly in three dimensions [8-10]. Indeed, this quantity has been argued to satisfy $F_{\mathbb{S}^{d}} \propto \mathcal{L}\left(f_{\infty}\right)$ for general highercurvature bulk theories, with the proportionality coefficient precisely agreeing with the one predicted by (9)—see, e.g., $[22,33]$. Hence, from the boundary CFT point of view, (9) tells us that the problem of computing $F_{\mathbb{S}_{\varepsilon}^{d}}$ for a given
Einstein-like theory, can actually be mapped to the one of evaluating the round $\mathbb{S}^{d}$ free energy for a different theory characterized by the same bulk Lagrangian, but different couplings $\tilde{\mu}_{n}$ such that $h\left(\tilde{f}_{\infty}\right)=0$ is satisfied for $\tilde{f}_{\infty} \equiv f_{\infty} /(1+\varepsilon)$.

An apparently similar connection between $\mathfrak{F}_{\mathbb{S}_{\varepsilon}^{d}}$ and $\mathfrak{F}_{\mathbb{S}^{d}}$ was found for $d=3, \mathcal{N}=2$ supersymmetric CFTs in Ref. [69]. However, supersymmetry requires additional background fields to be turned on besides the metric, which makes the corresponding free energies $\mathfrak{F}_{\mathbb{S}_{\varepsilon}^{d}}$ inequivalent from our $F_{\mathbb{S}_{\varepsilon}^{d}}$ [2]. Besides, the independence on the squashing parameter is generally true in the supersymmetric case, but not for $F_{\mathbb{S}_{\varepsilon}^{d}}$.

Back to the implications of Eq. (9), we know that the round sphere is a local extremum for the function $F_{\mathbb{S}_{\varepsilon}^{d}}$ [2], namely, $d F_{\mathbb{S}_{\varepsilon}^{d}} /\left.d \varepsilon\right|_{\varepsilon=0} \equiv F_{\mathbb{S}_{\varepsilon}^{d}}^{\prime}(0)=0$ for general theories. This is also nicely implemented in Eq. (9). Indeed, comparing with Eq. (3), it is straightforward to show that, according to Eq. (9), $F_{\mathbb{S}_{\varepsilon}^{d}}^{\prime}(0) \propto h\left(f_{\infty}\right)$, which of course vanishes by definition, as $h\left(f_{\infty}\right)=0$ is nothing but the embedding condition of $\operatorname{AdS}_{(d+1)}$ on the corresponding theory. Furthermore, we know that $F_{S_{\varepsilon}^{d}}^{\prime \prime}(0)$ is fully determined by the stress-tensor two-point function charge $C_{T}$ for general odd-dimensional CFTs [2]. In particular, for $d=3$ and $d=5$, it was found (in our conventions) that [70]

$$
F_{S_{\varepsilon}^{3}}^{\prime \prime}(0)=-\frac{\pi^{4}}{3} C_{T}, \quad F_{S_{\varepsilon}^{5}}^{\prime \prime}(0)=+\frac{\pi^{6}}{15} C_{T} .
$$

Now, using Eqs. (3), (5), and (9), we find, after some manipulations,

$$
F_{\mathbb{S}_{\varepsilon}^{d}}^{\prime \prime}(0)=\frac{(-1)^{[(d-1) / 2]} \pi^{d+1}(d-1)^{2}}{2 d !} C_{T}
$$

This expression reduces to the general results in Eq. (10), which is another highly nontrivial check of Eq. (9). Interestingly, it provides a generalization of the universal connection between $F_{S_{s}^{d}}^{\prime \prime}(0)$ and $C_{T}$, which must hold for general odd-dimensional CFTs (holographic or not).

Universal expansion in the squashing parameter.-As we have seen, the leading term in the $\varepsilon \rightarrow 0$ expansion of $F_{\mathbb{S}_{\varepsilon}^{d}}$ is quadratic in the deformation, and proportional to the stress-tensor two-point function charge $C_{T}$ for general CFTs. A question left open in Ref. [2] was the possibility that the subleading term, cubic in $\varepsilon$, could present an analogous universal behavior, in the sense of being fully characterized by the corresponding three-point function charges. Since $\varepsilon$ encodes a metric deformation, one expects $F_{\mathbb{S}_{\varepsilon}^{d}}^{(n)}$ to involve integrated $n$-point functions of the stress tensor. For general parity-even three-dimensional CFTs, the corresponding three-point function is completely fixed by conformal symmetry up to two theory-dependent quantities 
[51]. These can be chosen to be $C_{T}$, plus an additional dimensionless quantity, customarily denoted $t_{4}$ [76]. Hence, we expect a linear combination of $C_{T}$ and $C_{T} t_{4}$ to appear in the $\mathcal{O}\left(\varepsilon^{3}\right)$ term. The analysis in Ref. [2] shows however that, besides these contributions, an additional correlator of the form $\langle(\delta T / \sqrt{g} \delta g) T\rangle$-which depends on additional details of the specific CFT - appears at that order for general metric perturbations. The possibility that this term does not really contribute for certain metric perturbations, including our class of squashings, was left open.

The available partial results-numerical for a free scalar and a free fermion, and analytic for holographic Einstein gravity-did not suffice to provide a conclusive answer. In particular, the exact result for the free energy in holographic Einstein gravity is a polynomial of order 2 in $\varepsilon$, namely, $F_{\mathbb{S}_{\varepsilon}^{3}}^{\mathrm{E}}=\pi L^{2}\left(1-\varepsilon^{2}\right) /(2 G)$, which means that its Taylor expansion around $\varepsilon=0$ is trivial, and precisely ends with the quadratic piece-which is of course controlled by $C_{T}$ in agreement with Eq. (10), as can be readily verified using Eq. (6).

Happily, the new Taub-NUT solutions constructed in Ref. [56] for Einsteinian cubic gravity provide us with an additional family of holographic models for which we can access the cubic contribution, and explore its possible universality by testing it against the free-field numerics.

Using the result obtained in Ref. [33] for $t_{4}$ in holographic Einsteinian cubic gravity, we can express the squashed-sphere free energy of the corresponding dual theory for small values of $\varepsilon$ as

$$
F_{\mathbb{S}_{\varepsilon}^{3}}=F_{\mathbb{S}_{0}^{3}}-\frac{\pi^{4} C_{T}}{6} \varepsilon^{2}\left[1-\frac{t_{4}}{630} \varepsilon+\mathcal{O}\left(\varepsilon^{2}\right)\right],
$$

where the holographic mapping between boundary and bulk quantities is given by $F_{\mathbb{S}_{0}^{3}}^{\mathrm{ECG}}=\left(1+3 \mu f_{\infty}^{2}\right) \pi L^{2} /$ $\left(2 f_{\infty} G\right), C_{T}^{\mathrm{ECG}}=\left(1-3 \mu f_{\infty}^{2}\right) 3 L^{2} /\left(\pi^{3} f_{\infty} G\right)$, and $C_{T}^{\mathrm{ECG}} t_{4}^{\mathrm{ECG}}=$ $-3780 \mu f_{\infty} L^{2} /\left(\pi^{3} G\right)$, which naturally reduce to the Einstein gravity results in the $\mu \rightarrow 0$ limit.

The leading correction to the round-sphere result agrees with the general result [Eq. (10)], as it should. But now we have a nontrivial subleading piece, cubic in $\varepsilon$, and proportional to $C_{T} t_{4}$. As we mentioned above, it is not obvious that this term should not depend on additional theorydependent quantities on general grounds. Luckily, we can use the numerical free-field results in Ref. [2] to perform two highly nontrivial tests of the possible validity of Eq. (12) beyond holography. In order to do so, we study the function

$$
T(\varepsilon) \equiv \frac{630}{\varepsilon}\left[1+\frac{6\left(F_{\mathbb{S}_{\varepsilon}^{3}}-F_{\mathbb{S}_{0}^{3}}\right)}{\pi^{4} C_{T} \varepsilon^{2}}\right]
$$

for the conformally coupled scalar (s) and the free Dirac fermion (f) free energies near $\varepsilon=0$. Naturally, if Eq. (12)

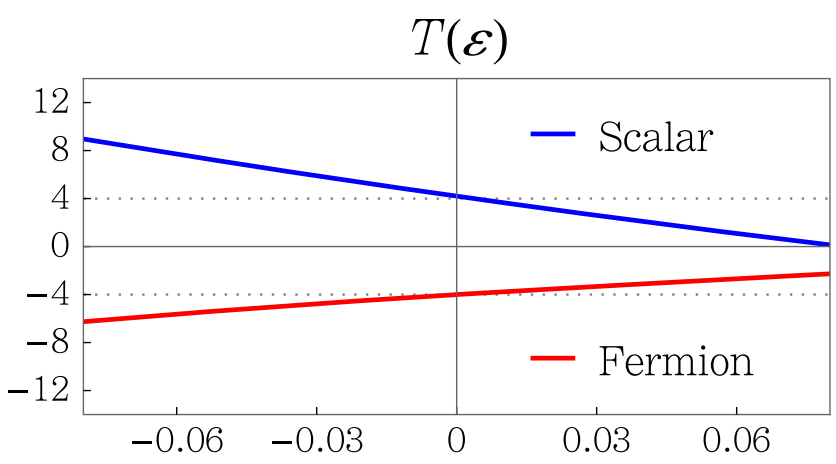

FIG. 1. We plot the function $T(\varepsilon)$ defined in (13) near $\varepsilon=0$ for a free scalar (blue) and a free fermion (red) using the numerical results for $F_{\mathbb{S}_{\varepsilon}^{3}}^{\text {s,f }}$ obtained in Ref. [2]. $T(\varepsilon=0)=t_{4}$ is satisfied in both cases with high accuracy, which provides strong evidence in favor of the conjectural general expression [Eq. (12)].

held for these theories, we should obtain $T(\varepsilon=0)=t_{4}$ which, for the scalar and the fermion, are respectively given by $t_{4}^{\mathrm{s}}=+4$ and $t_{4}^{\mathrm{f}}=-4[29,51]$. The result of this analysis is shown in Fig. 1, where it is manifest that this is precisely satisfied in both cases-details on the numerical methods utilized in the computation of $F_{\mathbb{S}_{\varepsilon}^{3}}^{\mathrm{s}}, F_{\mathbb{S}_{\varepsilon}^{3}}^{\mathrm{f}}$, and $T(\varepsilon)$ can be found in the Supplemental Material [49], which also includes Refs. [77-83]. The extremely different nature of the theories and techniques used in deriving the holographic and free-field results make us think that this property extends to arbitrary CFTs.

Conjecture: For general three-dimensional CFTs, the subleading term in the squashing-parameter $\varepsilon$ expansion of the free energy $F_{\mathbb{S}_{\varepsilon}^{3}}$ is universally controlled by the coefficient $t_{4}$ in the three-point function of the stress tensor. In particular, we conjecture that Eq. (12) holds for general theories.

The level of evidence provided here in favor of Eq. (12) - involving free-field and holographic higherorder gravity calculations-is very similar to the one initially presented in Refs. [23,24] concerning the universal relation between the entanglement entropy of almostsmooth corner regions and the charge $C_{T}$, which was eventually proven for general CFTs in Refs. [84,85].

If our conjecture is true, an analogous expression should hold for higher odd-dimensional squashed spheres. In that case, one would expect the $\mathcal{O}\left(\varepsilon^{3}\right)$ term to be controlled by some combination of $C_{T}, t_{4}$ and the additional stress-tensor three-point function charge, $t_{2}$, which is nonvanishing for $d>3$.

Final comments.-In spite of the compelling evidence presented here in favor of our new conjectural relation [Eq. (12)], performing additional checks would be very desirable. In particular, this could be further tested using the holographic duals of the set of higher-order theories constructed in Ref. [87]. A more ambitious (and challenging) endeavor would be to prove it for general CFTs using 
field-theoretical techniques along the lines of Ref. [2]. For this, one would need to explain why the $\langle(\delta T / \sqrt{g} \delta g) T\rangle$ correlator mentioned above makes no contribution in this case.

The applicability of Eq. (9) is of course more restrictive, as it holds only for a specific class of holographic theories. Proving it in general for such theories would also be interesting though.

Put together, Eqs. (9) and (12) would have additional consequences in the holographic context. As one can easily check, $F_{S_{e}^{d}}^{(n)}(0)$ can always be written in terms of $(n-1)$ th (and lower) derivatives of $h\left(f_{\infty}\right)$. For example, one finds

$$
\begin{aligned}
F_{\mathbb{S}_{\varepsilon}^{d}}^{(3)}(0)= & \frac{(-1)^{[(d+1) / 2]} \pi^{d / 2}\left(d^{2}-1\right) L^{d-1}}{16 \Gamma\left[\frac{d}{2}\right] f_{\infty}^{[(d-1) / 2]} G} \\
& \times\left[(d-3) h^{\prime}\left(f_{\infty}\right)-f_{\infty} h^{\prime \prime}\left(f_{\infty}\right)\right] .
\end{aligned}
$$

Then, if Eq. (12) holds for general theories, it follows that for any $d=3$ holographic higher-order gravity of the Einstein-like class [88],

$$
t_{4}=210 f_{\infty} \frac{h^{\prime \prime}\left(f_{\infty}\right)}{h^{\prime}\left(f_{\infty}\right)} .
$$

Hence, one would be able to obtain the coefficient $t_{4}$ by taking a couple of derivatives of $h\left(f_{\infty}\right)$. This represents a dramatic simplification with respect to the standard holographic calculations involving energy fluxes-see, e.g., Refs. $[29,30,76]$. It is natural to expect that this formula generalizes to higher dimensions. In that case, we expect an expression of the form

$$
a_{(d)} t_{2}+b_{(d)} t_{4}=f_{\infty} \frac{h^{\prime \prime}\left(f_{\infty}\right)}{h^{\prime}\left(f_{\infty}\right)},
$$

to hold for general Einstein-like theories in arbitrary dimensions, for some dimension-dependent constants $a_{(d)}$ and $b_{(d)}$. Using the available results for $t_{2}$ and $t_{4}$ in $d=4$ quasitopological gravity [30] and $d \geq 4$ GaussBonnet [29], it is straightforward to set the following: $b_{(4)}=-1 / 21$ and $a_{(d)}=-(d-2)(d-3) /[2 d(d-1)]$. In fact, a formula equivalent to Eq. (16) valid in the particular case of Lovelock theories-for which $t_{4}=0$ - was shown to be true in Refs. [32,53] for the same value of $a_{(d)}$. This provides additional support for the validity of Eq. (16) for general Einstein-like theories. It would be interesting to test the validity for such additional theories in various dimensions and, if correct in general, to determine the value of $b_{(d \geq 5)}$.

$h^{(n)}\left(f_{\infty}\right)$ appears to be related to the $(n+1)$-point function of the boundary stress tensor, therefore acting as some sort of generating functional. Interestingly, the "zero-point function" corresponding to the regularized round-sphere free energy $F_{\mathbb{S}^{d}}$ also satisfies this pattern, as it can be extracted from an integral involving $h\left(f_{\infty}\right)$, namely [89]

$$
\begin{aligned}
F_{\mathbb{S}^{d}}= & \frac{(-1)^{[(d+1) / 2]} \pi^{d / 2}(d+1)(d-1) L^{d-1}}{16 \Gamma\left[\frac{d}{2}\right] G} \\
& \times \int^{f_{\infty}} \frac{h(x)}{x^{[(d+3) / 2]}} d x .
\end{aligned}
$$

Integrating by parts in this expression, and using Eqs. (5) and (6), it is possible to find the suggestive relation [90]

$$
C_{T}=\frac{(-1)^{[(d-1) / 2]} \Gamma[d+2]}{\pi^{d+1}(d-1)^{2}} f_{\infty}\left[\frac{\partial F_{\mathbb{S}^{d}}}{\partial f_{\infty}}\right],
$$

which is equivalent to the one recently found in Ref. [91], and which, for this class of theories, connects two seemingly unrelated quantities, such as $C_{T}$ and $F_{\mathbb{S}^{d}}$ [92].

We would like to thank Nikolay Bobev, Alejandro Ruipérez, and Yannick Vreys for useful discussions and comments. The work of P. B. was supported by a postdoctoral fellowship from the National Science Foundation of Belgium (FWO). The work of P. A.C. is funded by Fundación la Caixa through a "la Caixa-Severo Ochoa" International predoctoral grant and partially by the MINECO/FEDER, UE Grant No. FPA2015-66793-P, and the "Centro de Excelencia Severo Ochoa" Program Grant No. SEV-2016-0597. R. B. M. and R. A. H. were supported in part by the Natural Sciences and Engineering Research Council of Canada.

[1] V. Pestun et al., J. Phys. A 50, 440301 (2017).

[2] N. Bobev, P. Bueno, and Y. Vreys, J. High Energy Phys. 07 (2017) 093.

[3] S. Fischetti and T. Wiseman, J. High Energy Phys. 12 (2017) 133.

[4] D. Anninos, F. Denef, and D. Harlow, Phys. Rev. D 88, 084049 (2013).

[5] G. Conti, T. Hertog, and Y. Vreys, J. High Energy Phys. 09 (2018) 068.

[6] T. Hertog, G. Tartaglino-Mazzucchelli, T. Van Riet, and G. Venken, J. High Energy Phys. 02 (2018) 024.

[7] S. W. Hawking and T. Hertog, J. High Energy Phys. 04 (2018) 147.

[8] I. R. Klebanov, S. S. Pufu, and B. R. Safdi, J. High Energy Phys. 10 (2011) 038.

[9] H. Casini and M. Huerta, Phys. Rev. D 85, 125016 (2012).

[10] S. S. Pufu, J. Phys. A 50, 443008 (2017).

[11] In particular, Eq. (1) preserves an $\mathrm{SU}(d+1 / 2) \times \mathrm{U}(1)$ subgroup of the usual $\mathrm{SO}(d+1)$ preserved by the usual round-sphere metric in $d$ dimensions.

[12] S. W. Hawking, C. J. Hunter, and D. N. Page, Phys. Rev. D 59, 044033 (1999).

[13] J. S. Dowker, Classical Quantum Gravity 16, 1937 (1999).

[14] A. Chamblin, R. Emparan, C. V. Johnson, and R. C. Myers, Phys. Rev. D 59, 064010 (1999). 
[15] R. Emparan, C. V. Johnson, and R. C. Myers, Phys. Rev. D 60, 104001 (1999)

[16] S. A. Hartnoll and S. P. Kumar, J. High Energy Phys. 06 (2005) 012.

[17] N. Bobev, T. Hertog, and Y. Vreys, J. High Energy Phys. 11 (2016) 140.

[18] J. M. Maldacena, Int. J. Theor. Phys. 38, 1113 (1999); Adv. Theor. Math. Phys. 2, 231 (1998).

[19] E. Witten, Adv. Theor. Math. Phys. 2, 253 (1998).

[20] S. S. Gubser, I. R. Klebanov, and A. M. Polyakov, Phys. Lett. B 428, 105 (1998).

[21] R. C. Myers and A. Sinha, Phys. Rev. D 82, 046006 (2010).

[22] R. C. Myers and A. Sinha, J. High Energy Phys. 01 (2011) 125.

[23] P. Bueno, R. C. Myers, and W. Witczak-Krempa, Phys. Rev. Lett. 115, 021602 (2015).

[24] P. Bueno and R. C. Myers, J. High Energy Phys. 08 (2015) 068.

[25] M. Mezei, Phys. Rev. D 91, 045038 (2015).

[26] C.-S. Chu and R.-X. Miao, J. High Energy Phys. 12 (2016) 036.

[27] D. J. Gross and J. H. Sloan, Nucl. Phys. B291, 41 (1987).

[28] X. O. Camanho and J. D. Edelstein, J. High Energy Phys. 06 (2010) 099.

[29] A. Buchel, J. Escobedo, R. C. Myers, M. F. Paulos, A. Sinha, and M. Smolkin, J. High Energy Phys. 03 (2010) 111 .

[30] R. C. Myers, M. F. Paulos, and A. Sinha, J. High Energy Phys. 08 (2010) 035.

[31] J. de Boer, M. Kulaxizi, and A. Parnachev, J. High Energy Phys. 06 (2010) 008.

[32] X. O. Camanho, J. D. Edelstein, and J. M. Sánchez de Santos, Gen. Relativ. Gravit. 46, 1637 (2014).

[33] P. Bueno, P. A. Cano, and A. Ruipérez, J. High Energy Phys. 03 (2018) 150.

[34] P. Bueno, P. A. Cano, V. S. Min, and M. R. Visser, Phys. Rev. D 95, 044010 (2017).

[35] The special case $n=(d+1) / 2$ is automatically excluded from the sum, as no invariant of that order contributes to the vacua equation.

[36] Namely, we restrict to those for which the linearized equations take the form $G_{a b}^{\mathrm{L}}=8 \pi G_{\text {eff }} T_{a b}$, where $G_{a b}^{\mathrm{L}}$ is the linearized Einstein tensor, $T_{a b}$ is some possible matter stress tensor, and $G_{\text {eff }}$ is the effective Newton constant.

[37] D. Lovelock, Aequationes mathematicae 4, 127 (1970).

[38] D. Lovelock, J. Math. Phys. (N.Y.) 12, 498 (1971).

[39] P. Bueno, P. A. Cano, A. O. Lasso, and P. F. Ramirez, J. High Energy Phys. 04 (2016) 028.

[40] J. Oliva and S. Ray, Classical Quantum Gravity 27, 225002 (2010).

[41] R. C. Myers and B. Robinson, J. High Energy Phys. 08 (2010) 067.

[42] M. H. Dehghani, A. Bazrafshan, R. B. Mann, M. R. Mehdizadeh, M. Ghanaatian, and M. H. Vahidinia, Phys. Rev. D 85, 104009 (2012).

[43] A. Cisterna, L. Guajardo, M. Hassaine, and J. Oliva, J. High Energy Phys. 04 (2017) 066.

[44] P. Bueno and P. A. Cano, Phys. Rev. D 94, 104005 (2016).

[45] R. A. Hennigar, D. Kubiznak, and R. B. Mann, Phys. Rev. D 95, 104042 (2017).
[46] A. Karasu, E. Kenar, and B. Tekin, Phys. Rev. D 93, 084040 (2016).

[47] Y.-Z. Li, H.-S. Liu, and H. Lu, J. High Energy Phys. 02 (2018) 166.

[48] Y.-Z. Li, H. Lu, and J.-B. Wu, Phys. Rev. D 97, 024023 (2018).

[49] See Supplemental Material at http://link.aps.org/ supplemental/10.1103/PhysRevLett.122.071602 for additional calculations supporting the results presented in the main text.

[50] Conformal invariance completely constrains the correlator $\left\langle T_{\mu \nu}(x) T_{\lambda \rho}(0)\right\rangle$ up to a theory-dependent quantity, customarily denoted $C_{T}$, as $\left\langle T_{\mu \nu}(x) T_{\lambda \rho}(0)\right\rangle=$ $C_{T} \mathcal{I}_{\mu \nu, \lambda \rho}(x) /|x|^{2 d}$, where $\mathcal{I}_{\mu \nu, \lambda \rho}$ is a fixed dimensionless tensor structure [51].

[51] H. Osborn and A. C. Petkou, Ann. Phys. (N.Y.) 231, 311 (1994).

[52] Equation (5) was previously proven in the particular case of Lovelock theories in Refs. [32,53].

[53] X. O. Camanho, J. D. Edelstein, and M. F. Paulos, J. High Energy Phys. 05 (2011) 127.

[54] Observe that our convention for $C_{T}$ differs from that in Ref. [2] by a factor $1 / \mathcal{S}_{d}^{2}=\Gamma[d / 2]^{2} /\left(4 \pi^{2}\right)$. It agrees, however, with the convention in Refs. [22,24,29,30]. Also note that it is customary to write Einstein gravity results in terms of $L / \sqrt{f_{\infty}}$, instead of $L$ alone. This is irrelevant for Einstein gravity itself, for which $f_{\infty}=1$, but needs to be kept in mind for higher-order theories.

[55] O. Aharony, S. S. Gubser, J. M. Maldacena, H. Ooguri, and Y. Oz, Phys. Rep. 323, 183 (2000).

[56] P. Bueno, P. A. Cano, R. A. Hennigar, and R. B. Mann, J. High Energy Phys. 10 (2018) 095.

[57] A. Awad and A. Chamblin, Classical Quantum Gravity 19, 2051 (2002).

[58] M. H. Dehghani and R. B. Mann, Phys. Rev. D 72, 124006 (2005).

[59] M. H. Dehghani and S. H. Hendi, Phys. Rev. D 73, 084021 (2006).

[60] S. H. Hendi and M. H. Dehghani, Phys. Lett. B 666, 116 (2008).

[61] D. Astefanesei, R. B. Mann, and E. Radu, J. High Energy Phys. 01 (2005) 049.

[62] R. Clarkson, A. M. Ghezelbash, and R. B. Mann, Int. J. Mod. Phys. A 19, 3987 (2004).

[63] C. O. Lee, Phys. Lett. B 670, 146 (2008).

[64] E. Shaghoulian, Phys. Rev. Lett. 119, 131601 (2017).

[65] V. Balasubramanian and P. Kraus, Commun. Math. Phys. 208, 413 (1999).

[66] Y. Brihaye and E. Radu, J. High Energy Phys. 09 (2008) 006.

[67] C. Teitelboim and J. Zanelli, Classical Quantum Gravity 4, L125 (1987).

[68] M. H. Dehghani and M. H. Vahidinia, Phys. Rev. D 84, 084044 (2011).

[69] N. Hama, K. Hosomichi, and S. Lee, J. High Energy Phys. 05 (2011) 014.

[70] Related expressions had been previously found in the context of $d=3, \mathcal{N}=2$ supersymmetric CFTs [71]—-see also Refs. [69,72-75]. A detailed discussion of the connection can be found in Sec. 5.1 of Ref. [2]. 
[71] C. Closset, T. T. Dumitrescu, G. Festuccia, and Z. Komargodski, J. High Energy Phys. 05 (2013) 017.

[72] C. Closset, T. T. Dumitrescu, G. Festuccia, Z. Komargodski, and N. Seiberg, J. High Energy Phys. 09 (2012) 091.

[73] C. Closset, T. T. Dumitrescu, G. Festuccia, Z. Komargodski, and N. Seiberg, J. High Energy Phys. 10 (2012) 053.

[74] Y. Imamura and D. Yokoyama, Phys. Rev. D 85, 025015 (2012).

[75] D. Martelli, A. Passias, and J. Sparks, Nucl. Phys. B864, 840 (2012).

[76] D. M. Hofman and J. Maldacena, J. High Energy Phys. 05 (2008) 012.

[77] C. W. Misner, J. Math. Phys. (N.Y.) 4, 924 (1963).

[78] G. W. Gibbons and S. W. Hawking, Phys. Rev. D 15, 2752 (1977).

[79] R. C. Myers, Phys. Rev. D 36, 392 (1987).

[80] J. Ahmed, R. A. Hennigar, R. B. Mann, and M. Mir, J. High Energy Phys. 05 (2017) 134.

[81] R. Clarkson, L. Fatibene, and R. B. Mann, Nucl. Phys. B652, 348 (2003).

[82] A. Khodam-Mohammadi and M. Monshizadeh, Phys. Rev. D 79, 044002 (2009).

[83] D. V. Vassilevich, Phys. Rep. 388, 279 (2003).

[84] T. Faulkner, R. G. Leigh, and O. Parrikar, J. High Energy Phys. 04 (2016) 088.

[85] In contrast to Eq. (12), however, the subleading term in the smooth-limit expansion of the corner entanglement entropy (quartic in the deformation) was later shown not to be generically controlled by the stress-tensor three-point function charges in Ref. [86].

[86] P. Bueno and W. Witczak-Krempa, Phys. Rev. B 93, 045131 (2016).

[87] P. Bueno and P. A. Cano, Phys. Rev. D 96, 024034 (2017).

[88] It is immediate to check that this expression yields the right $t_{4}$ for Einsteinian cubic gravity [Eq. (8)], for which $h\left(f_{\infty}\right)=1-f_{\infty}+\mu f_{\infty}^{3}$.

[89] In even-dimensional CFTs, this expression yields - up to a $2(-1)^{-1 / 2} / \pi$ factor-the coefficient of the universal logarithmic contribution to the corresponding round-sphere free energy, given by $(-1)^{[(d-2) / 2]} 4 a^{*}$, where $a^{*}$ is proportional to one of the trace-anomaly charges ( $a$ in $d=4$ ), e.g., Ref. [22].

[90] Let us note that there is an ambiguity in this expression, because there are many ways to express $F_{\mathbb{S}^{d}}$ as a function of $f_{\infty}$. There is a canonical form of $F_{\mathbb{S}^{d}}$ in which Eq. (18) works, and this form is $F_{\mathbb{S}^{d}}=F_{\mathbb{S}^{d}}^{\mathrm{E}}\left(1+\right.$ polynomial in $\left.f_{\infty}\right)$, where $F_{\mathbb{S}^{d}}^{\mathrm{E}}$ is the Einstein gravity result with the rescaled AdS scale $L / \sqrt{f_{\infty}}$.

[91] Y.-Z. Li, H. Lu, and Z.-F. Mai, J. High Energy Phys. 10 (2018) 063.

[92] In terms of $a^{*}$, the relation reads $C_{T}=-2 \Gamma[d+2] /$ $\left[\pi^{d}(d-1)^{2}\right] \cdot f_{\infty}\left[\partial a^{*} / \partial f_{\infty}\right]$, which is valid in general (even and odd) dimensions. 